\title{
Mitral valve repair and redo repair for mitral regurgitation in a heart transplant recipient
}

\author{
Wobbe Bouma1*, Johan Brügemann², Inez J Wijdh-den Hamer ${ }^{1}$, Theo J Klinkenberg ${ }^{1}$, Bart M Koene ${ }^{1}$, \\ Michiel Kuijpers ${ }^{1}$, Michiel E Erasmus ${ }^{1}$, Iwan CC van der Horst $^{2}$ and Massimo A Mariani ${ }^{1}$
}

\begin{abstract}
A 37-year-old man with end-stage idiopathic dilated cardiomyopathy underwent an orthotopic heart transplant followed by a reoperation with mitral annuloplasty for severe mitral regurgitation. Shortly thereafter, he developed severe tricuspid regurgitation and severe recurrent mitral regurgitation due to annuloplasty ring dehiscence. The dehisced annuloplasty ring was refixated, followed by tricuspid annuloplasty through a right anterolateral thoracotomy. After four years of follow-up, there are no signs of recurrent mitral or tricupid regurgitation and the patient remains in NYHA class II. Pushing the envelope on conventional surgical procedures in marginal donor hearts (both before and after transplantation) may not only improve the patient's functional status and reduce the need for retransplantation, but it may ultimately alleviate the chronic shortage of donor hearts.
\end{abstract}

Keywords: Heart transplantation, Mitral regurgitation, Mitral valve repair, Reoperation

\section{Background}

Although cardiac transplantation has become a relatively common procedure, its major limitation is still the chronic shortage of donor hearts. Potentially, the donor pool could be expanded by extending donor selection criteria and by performing conventional surgical procedures on the donor heart, such as coronary artery bypass grafting or valve repair [1-4]. The limits of these procedures are being pushed to improve the patient's functional status and to reduce the need for re-transplantation.

This report shows the feasibility of mitral valve repair and re-repair in a transplanted heart. To our knowledge, this is the first report of mitral valve re-repair in a transplanted heart.

\section{Case presentation}

A 37 -year-old man (length $1.98 \mathrm{~m}$, weight $120 \mathrm{~kg}$, body surface area $2.57 \mathrm{~m}^{2}$ ) with end-stage idiopathic dilated cardiomyopathy underwent an orthotopic heart transplant in June 2006. The donor was a 53-year-old man with no history of heart disease who had suffered

\footnotetext{
*Correspondence: w.bouma@umcg.nl

${ }^{1}$ Department of Cardiothoracic Surgery, University Medical Center Groningen, Groningen, the Netherlands

Full list of author information is available at the end of the article
}

intracerebral hemorrhage. A predonation echocardiogram revealed grade $1+$ mitral regurgitation (MR), no other valvular lesions, and a moderate left ventricular (LV) function (Table 1). Coronary angiography was unavailable. Palpation did not reveal any coronary artery disease.

The donor heart was preserved with St. Thomas' solution (flush-perfusion) and implanted using the biatrial anastomotic technique. The total ischaemic time of the donor heart was 226 minutes. Intraoperative transesophageal echocardiography (TEE) (during dopamine and noradrenaline support after weaning from cardiopulmonary bypass) revealed grade $1+\mathrm{MR}$. The postoperative course was complicated by prolonged inotropic support (3 days) and atrial fibrillation. The donor heart experienced substantial ischemic damage as shown by high levels of postoperative serum creatine kinase (maximum level $2500 \mathrm{U} / \mathrm{L}$ ) and creatine kinase-MB (maximum level $246 \mathrm{U} / \mathrm{L}$ ). A predischarge transthoracic echocardiogram (TTE) revealed grade $2+\mathrm{MR}$, grade $1+$ tricuspid regurgitation (TR), inferoposterior hypokinesia, and a moderate LV function. Sixteen routine endomyocardial biopsies in the first year after transplantation showed no significant rejection episodes (International Society of Heart and Lung Transplantation (ISHLT) grade $\leq$ IA).

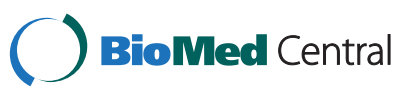


Table 1 Overview of events, treatment, NYHA class, and echocardiographic follow-up

\begin{tabular}{|c|c|c|c|c|c|}
\hline Event & Month \& year & Treatment & $\begin{array}{l}\text { Recipient } \\
\text { NYHA class }\end{array}$ & TTE/TEE & Echocardiographic findings \\
\hline Heart transplant & June 2006 & - & 4 & Pre-op donor TTE & MR $1+$, TR 0, moderate LVF \\
\hline Discharge & June 2006 & - & 3 & Pre-discharge TTE & $\begin{array}{l}\text { MR 2+, TR1+, moderate LVF } \\
\text { (inferoposterior hypokinesia) }\end{array}$ \\
\hline $\begin{array}{l}\text { NSTEMI } \\
\text { (inferoposterior) }\end{array}$ & June 2007 & $\begin{array}{l}\text { PCI RCA with } 2 \\
\text { PRO-kinetic stents }\end{array}$ & 3 & - & - \\
\hline \multirow[t]{3}{*}{ Follow-up } & January 2008 & - & 4 & TTE and TEE & $\begin{array}{l}\text { MR 4+ (Figure 1A), TR 2+, dilatation } \\
\text { of the LV and deterioration of LVF }\end{array}$ \\
\hline & & & & & $\begin{array}{l}\text { MR severity: jet surface area }\left(18.5 \mathrm{~cm}^{2}\right) / \\
\text { LA surface area }\left(36.5 \mathrm{~cm}^{2}\right)=51 \% \text {; vena } \\
\text { contracta }=65 \mathrm{~mm}\end{array}$ \\
\hline & & & & & 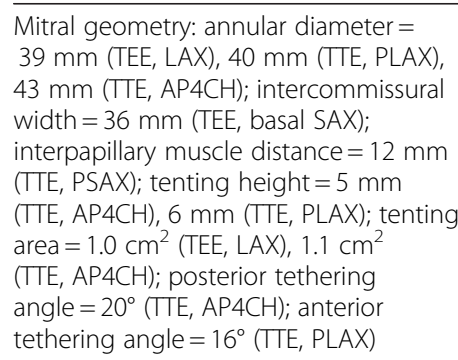 \\
\hline NSTEMI (inferior) & May 2008 & $\begin{array}{l}\text { Mitral valve repair } \\
\text { (CE classic } 32 \mathrm{~mm} \text { ring); } \\
\text { hybrid } \mathrm{PCl} \text { of the Cx}\end{array}$ & 2 & Post-op TTE & MR 1+, TR 1+, moderate LVF \\
\hline Total AV block & May 2008 & DDD-pacemaker & 2 & - & - \\
\hline \multirow[t]{6}{*}{ Follow-up } & June 2008 & - & 3 & TTE and TEE & $\begin{array}{l}\text { MR 4+ (ring dehiscence) (Figure 1B), } \\
\text { TR 4+ (Figure 1C), moderate LVF }\end{array}$ \\
\hline & July 2008 & $\begin{array}{l}\text { Redo mitral repair } \\
\text { (ring refixation) and } \\
\text { tricuspid repair } \\
\text { (CE classic } 36 \mathrm{~mm} \text { ring) }\end{array}$ & 2 & Post-op TTE & MR 1+, TR 1+, moderate LVF \\
\hline & August 2008 & - & 2 & $T T E$ & $\begin{array}{l}\text { Moderate LVF, intraventricular } \\
\text { dyssynchronia }\end{array}$ \\
\hline & September 2008 & Upgrade to CRT-D & 2 & - & - \\
\hline & July 2010 & - & 2 & TEE & $\begin{array}{l}\text { MR 1+ (Figure 1D), TR 1+ (Figure 1D), } \\
\text { moderate LVF }\end{array}$ \\
\hline & February 2012 & - & 2 & TTE & MR 1+, TR 1+, moderate LVF \\
\hline
\end{tabular}

AP4CH, apical four-chamber view; AV, atrioventricular; CE, Carpentier-Edwards; CRT-D, cardiac resynchronization therapy-defibrillator; Cx, circumflex coronary artery; DDD, dual chamber/dual demand; LA, left atrial; LV(F), left ventricular (function); MR, mitral regurgitation; NSTEMI, non-ST-segment elevation myocardial infarction; NYHA, New-York Heart Association; PCl, percutaneous coronary intervention; (P)LAX, (parasternal) long-axis view; (P)SAX, (parasternal) short-axis view; $\mathrm{RCA}$, right coronary artery; TEE, transesophageal echocardiography; TR, tricuspid regurgitation; TTE, transthoracic echocardiography.

In June 2007, the patient suffered an inferoposterior nonST-segment elevation myocardial infarction (NSTEMI). A percutaneous coronary intervention $(\mathrm{PCI})$ of the right coronary artery was performed with implantation of two Biotronik PRO-kinetic stents (Biotronik, Berlin, Germany). The circumflex coronary artery did not show any abnormalities. In January 2008, TTE and TEE revealed grade $4+$ central MR (Figure 1A), grade $2+\mathrm{TR}$, and a dilated LV with deterioration of the moderate LV function. Additional analysis of MR severity and mitral valve geometry is shown in Table 1. Mitral valve surgery was initially postponed, but in the following months complaints of dyspnea on exertion worsened and in May 2008 the patient suffered an inferior NSTEMI (this time based on a stenosis in the circumflex coronary artery). A hybrid treatment strategy was chosen with mitral valve surgery followed by PCI of the circumflex coronary artery.

In May 2008, mitral valve repair was performed through a redo median sternotomy. The mitral valve was exposed with a right atrial, trans-septal approach. No structural mitral valve abnormalities were found and saline testing revealed central regurgitation. MR was presumed to be ischaemic in etiology. After careful sizing, annular dilatation was corrected by implantation of an undersized $32 \mathrm{~mm}$ Carpentier-Edwards classic mitral valve annuloplasty ring with interrupted 2-0 Ticron sutures (i.e., a downsizing of two ring sizes). Postoperative recovery was complicated by 

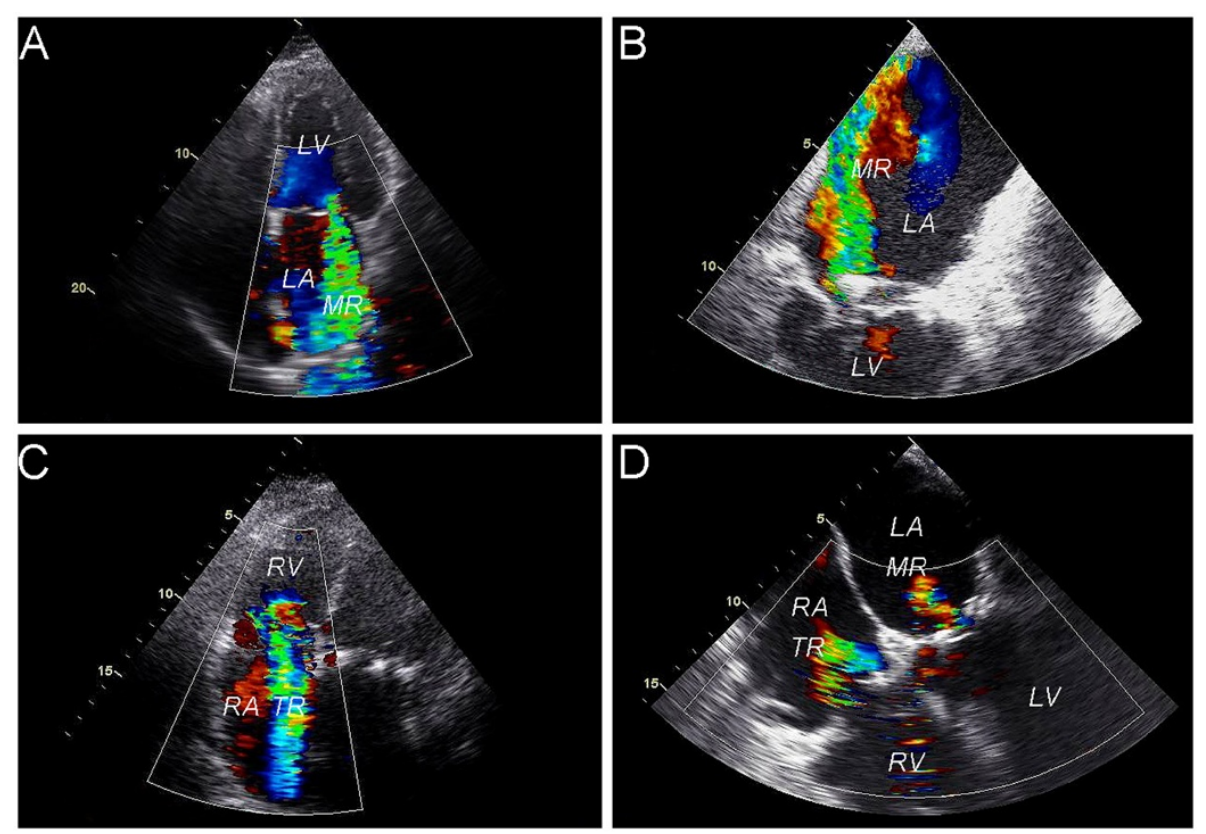

Figure 1 Doppler echocardiographic imaging. A. Preoperative TTE; grade 4+ MR. B. Postoperative TEE; grade 4+ recurrent MR along the posterior annulus. C. Postoperative TTE; grade 4+ TR. D. Two-year follow-up TEE; grade 1+ residual TR and MR. LA, left atrium; LV, left ventricle; $M R$, mitral regurgitation; RA, right atrium; RV, right ventricle; TEE, transesophageal echocardiography; $T R$, tricuspid regurgitation; TTE, transthoracic echocardiography.

a total atrioventricular (AV) block, which eventually required implantation of a DDD (dual chamber/dual demand)-pacemaker. On the seventh day after surgery, TTE showed grade $1+\mathrm{MR}$ and $\mathrm{TR}$ and a moderate LV function.

Follow-up TTE and TEE after six weeks showed severe recurrent $M R$ (grade 4+) along the posterior annulus (Figure 1B) and severe TR (grade 4+) (Figure 1C). In July 2008, a right anterolateral thoracotomy was performed. The mitral valve was again exposed with a right atrial, trans-septal approach. Inspection revealed mitral annuloplasty ring dehiscence along the posterior annulus. The annuloplasty ring was refixated with interrupted 2-0 Ticron pledgeted sutures. Inspection of the tricuspid valve revealed no structural abnormalities. A $36 \mathrm{~mm}$ Carpentier-Edwards classic tricuspid annuloplasty ring was implanted. On the fifth day after surgery, TTE showed grade $1+\mathrm{MR}$ and $\mathrm{TR}$ and a moderate LV function.

In August 2008, TTE still showed a moderate LV function with evidence of intraventricular dyssynchronia. In September 2008, the DDD-pacemaker was upgraded to CRT-D (cardiac resynchronization therapy-defibrillator). In July 2010 (after a follow-up of two years) (Figure 1D) and in February 2012 (after a follow-up of nearly four years), MR and TR remained grade $1+, L V$ function remained moderate, and the patient remained in NYHA functional class II.

\section{Discussion}

The reported experience with repair of atrioventricular valves after cardiac transplantation is still limited. Publications include different reports of single or double atrioventricular valve repair, either before (ex vivo bench surgery) or after transplantation [1-4]. However, this case is the first report of mitral valve re-repair in a transplanted heart.

Atrioventricular valve regurgitation after heart transplantation is common [1]. Early after transplantation, regurgitation is attributable to edema and poor lymphatic drainage, which normally subsides after about three months $[1,4]$. Some authors assume that the biatrial anastomosis results in atrioventricular size mismatch or malalignment, which lies at the basis of annular dilatation and valve dysfunction [1]. The bicaval anastomosis has been shown to preserve atrial geometry and size, and may be associated with reduced atrioventricular valve dysfunction [5]. Progression of natural valve disease in the donor heart at the time of transplantation may also cause atrioventricular valve regurgitation [1]. Endomyocardial biopsy-induced chordal damage is a known cause of TR [1] and accelerated graft atherosclerosis may lead to ischemic valve disease $[1,3]$.

The etiology in this case is most likely chronic ischaemic MR (CIMR); the donor heart was subjected to a relatively long ischemic time, there was a documented inferoposterior myocardial infarction, inferoposterior 
hypokinesia, LV dilatation, and no structural mitral valve abnormality [6]. The posterior part of the mitral valve annulus has been shown to experience the largest amounts of mechanical strain during the cardiac cycle [7]. In addition, the biatrial method may have contributed to distortion of the atrial geometry with increased strain levels on the posterior annulus. Large amounts of strain on the sutures of the posterior part of the annuloplasty ring may eventually have caused dehiscence. Careful analysis of preoperative tenting parameters (Table 1) showed values well below the cutoff values of independent predictors of annuloplasty failure [6]. Since initial mitral annuloplasty was justified based on these parameters, we chose to perform mitral valve re-repair. Initial mitral valve annuloplasty with a more flexible ring (e.g., the Carpentier-Edwards Physio II ring) or a ring specifically tailored for CIMR (e.g., the Edwards GeoForm ring) might have reduced the chance of annuloplasty dehiscence. At the time, however, these rings were not yet in use or available on the market. The etiology of TR in this case remains uncertain. Since no structural abnormalities were found, it may be related to atrioventricular size mismatch, progression of natural valve disease, or a combination of both.

\section{Conclusions}

In conclusion, double atrioventricular valve repair (including mitral valve re-repair) can be performed with good mid-term results after orthotopic heart transplantation. Acceptance of marginal donor hearts may lead to a complicated and demanding postoperative course, as shown in this case. However, pushing the envelope on convential surgical procedures in the transplanted heart may not only improve the patient's functional status and reduce the need for retransplantation, but it may ultimately alleviate the chronic shortage of donor hearts.

\section{Consent}

Written informed consent was obtained from the patient for publication of this case report and any accompanying images. A copy of the written consent is available for review by the Editor-in-Chief of this journal.

\section{Competing interests}

The authors declare that they have no competing interests.

\section{Authors' contributions}

WB and JB collected the data and wrote the manuscript. IW, TK, BK, MK, ME, $\mathrm{H}$, and MM participated in the design of the manuscript and they revised and critically reviewed the manuscript. All authors read and approved the final manuscript.

\section{Acknowledgements}

This study was financially supported by University Medical Center Groningen and the Groningen University Institute for Drug Exploration.

\section{Author details}

'Department of Cardiothoracic Surgery, University Medical Center Groningen, Groningen, the Netherlands. ${ }^{2}$ Department of Cardiology, University Medical Center Groningen, Groningen, the Netherlands.

Received: 24 February 2012 Accepted: 23 September 2012 Published: 29 September 2012

\section{References}

1. Fernandez J, Babadjanov D, Farivar RS: Mitral and tricuspid valve repair 21 years after cardiac transplantation. J Thorac Cardiovasc Surg 2010, 140:e3-e4.

2. Prieto $D$, Antunes $P$, Antunes MJ: Donor mitral valve repair in cardiac transplantation. Transplant Proc 2009, 41:932-934.

3. Wigfield $\mathrm{CH}$, Lewis A, Parry G, Dark JH: Mitral valve dysfunction and repair following orthotopic heart transplantation: a case report. Transplant Proc 2008, 40:1796-1797.

4. Wijburg ER, Balk AHMM, van Herwerden LA: Double valve repair in a transplanted heart. J Thorac Cardiovasc Surg 1998, 115:250-251.

5. Schnoor M, Schäfer T, Lühmann D, Sievers HH: Bicaval versus standard technique in orthotopic heart transplantation: a systematic review and meta-analysis. J Thorac Cardiovasc Surg 2007, 134:1322-1331.

6. Bouma W, van der Horst IC, Wijdh-den Hamer IJ, Erasmus ME, Zijlstra F, Mariani MA, Ebels T: Chronic ischaemic mitral regurgitation. Current treatment results and new, mechanism-based surgical approaches. Eur J Cardiothorac Surg 2010, 37:170-185.

7. Eckert CE, Zubiate B, Vergnat M, Gorman JH 3rd, Gorman RC, Sacks MS: In vivo dynamic deformation of the mitral valve annulus. Ann Biomed Eng 2009, 37:1757-1771.

doi:10.1186/1749-8090-7-100

Cite this article as: Bouma et al: Mitral valve repair and redo repair for mitral regurgitation in a heart transplant recipient. Journal of Cardiothoracic Surgery 2012 7:100.

\section{Submit your next manuscript to BioMed Central and take full advantage of:}

- Convenient online submission

- Thorough peer review

- No space constraints or color figure charges

- Immediate publication on acceptance

- Inclusion in PubMed, CAS, Scopus and Google Scholar

- Research which is freely available for redistribution 